\title{
Complications associated with laparoscopic sleeve gastrectomy - a review
}

\author{
Paulina Woźniewska, Inna Diemieszczyk, Hady Razak Hady \\ $1^{\text {st }}$ Department of General and Endocrine Surgery, University Hospital of Bialystok, Bialystok, Poland
}

Gastroenterology Rev 2021; 16 (1): 5-9

DOI: https://doi.org/10.5114/pg.2021.104733

Key words: laparoscopic sleeve gastrectomy, bariatric surgery.

Address for correspondence: Paulina Woźniewska MD, $1^{\text {st }}$ Department of General and Endocrine Surgery, University Hospital of Bialystok, Bialystok, Poland, e-mail: pwozniewska@gmail.com

\begin{abstract}
Laparoscopic sleeve gastrectomy (LSG) is an essential bariatric procedure performed in obese patients, which provides significant weight loss and has a positive impact on obesity-related diseases. However, as with any surgical procedure, it carries the risk of complications. The complications that can arise in patients following LSG are divided into acute (diagnosed within 30 days after the surgery) and late. Early complications that require rapid management include haemorrhage (intraluminal or extraluminal), leak in the staple line, and abscess formation. Late complications include gastric stenosis, nutrient deficiencies, mediastinal pouch migration, and the development or exacerbation of gastroesophageal reflux diseases. In this review, we present the basic information about most common complications following LSG, and their symptoms, diagnostic tools, and management.
\end{abstract}

\section{Introduction}

Obesity is one of the major health care issues of the $21^{\text {st }}$ century throughout the world. According to World Health Organization data in 2016, over 650 million adults have struggled with obesity, which constitutes $13 \%$ of the world population [1]. Progressive body mass gain leads to gradual failure of organs and systems. Abdominal obesity is considered to be the dominant risk factor for developing metabolic syndrome, which includes hypertension, dyslipidaemia, and insulin resistance $[2,3]$. Patients diagnosed with metabolic syndrome more often develop myocardial infarction, stroke, and type 2 diabetes, which significantly increases the cardiovascular risk and contributes to increased mortality in comparison to the general population $[4,5]$.

The treatment of obesity is based on restoring the balance between food intake and its expenditure, as well as taking care of obesity-related comorbidities. Conservative treatment of obesity includes increased physical activity, dietary changes, and pharmacological therapy. However, these methods are often insufficient to obtain satisfactory weight loss, and so patients are offered bariatric surgery. Nowadays the most commonly performed bariatric procedures are laparoscopic sleeve gastrectomy (LSG), Roux-en-Y gastric by-pass (LRYGB), and implementation of an adjustable gastric band (laparoscopic adjustable gastric banding - LAGB) [6]. The register kept by the International Federation for the Surgery of Obesity and Metabolic Disorders (IFSO) has noted 394,431 bariatric procedures performed in 51 countries since 2014 . The most frequently performed surgery has been laparoscopic sleeve gastrectomy, which constitutes $46.0 \%$ of all interventions [7]. LSG is currently considered to be the most effective treatment solution for morbid obesity, which leads not only to achieve significant and permanent weight loss, but also to partial or total remission of obesity-related comorbidities [8, 9].

Sleeve gastrectomy was introduced in 1990 as an alternative method for distal stomach resection with duodenal switch to reduce the complication rate [10]. The first laparoscopic procedure took place in 1999 and was conducted by Ren et al. [11]. Since then, sleeve gastrectomy has been gaining popularity as a method of obesity treatment and as a subject of medical research [12]. LSG is a procedure in which the greater curvature is resected, reducing the volume of the stomach by about $80 \%$, which leads to significant limitation of food intake. Additionally, removal of the stomach fundus decreases the number of cells producing ghrelin - the "hunger hormone". The reduction in plasma 
ghrelin concentration promotes the feeling of satiety and restrains food intake [13].

Despite the fact that metabolic-bariatric surgery is currently the most effective method of morbid obesity treatment, the surgical interventions are still associated with a risk of perioperative complications. According to the IFSO, the incidence of postoperative complications for LSG is $2.12 \%$, which is lower than for LRYGB (3.02\%) [14]. The mortality rate for LSG varies from $0.18 \%$ to $0.27 \%$ and depends on age, sex, comorbidities, and the references of the centre where the surgery is performed [15].

\section{Review}

Complications after LSG may be divided into early (acute), which develop within 30 days after the surgery, and late, that occur over 30 days after the surgical procedure. The most common complications after LSG are leakage, bleeding in the staple line, gastric pouch stenosis, mediastinal pouch migration, wound infection, and nutrient deficiencies [16]. Less frequently acute pancreatitis, partial spleen infarction, and pulmonary embolism are encountered. A summary of the possible complications occurring after LSG is presented in Table I.

\section{Early complications}

Bleeding is the most frequent complication occurring after LSG, which is observed in $1.16-4.94 \%$ of all cases [17]. It is mainly formed in the staple line; however, it may also be caused by incorrect coagulation of blood vessels, damage of parenchymal organs (spleen and liver), or disturbances in haemostasis. Gastrointestinal haemorrhage is usually caused by bleeding from the staple line. Patients present symptoms of upper gastrointestinal bleeding such as haematemesis and melena. In turn, bleeding into the peritoneal cavity manifests as tachycardia, hypotension, and decreased haemoglobin. The source of peritoneal haemorrhage is

Table I. Complications associated with laparoscopic sleeve gastrectomy

\begin{tabular}{lc}
\multicolumn{1}{c}{$\begin{array}{c}\text { Early complications } \\
\text { (<30 days })\end{array}$} & $\begin{array}{c}\text { Late complications } \\
(>\mathbf{3 0} \text { days })\end{array}$ \\
\hline Haemorrhage & Gastric stenosis \\
\hline Staple line leak & Nutrient deficiency \\
\hline Intraabdominal abscess & Gastroesophageal reflux \\
& disease \\
\hline Wound infection & Trocar-related hernia \\
Acute pancreatitis & Mediastinal pouch migration \\
Pulmonary embolism & Mental health issues \\
Thrombophlebitis & Eating disorders \\
Rhabdomyolysis & \\
Acute kidney injury (AKI) & \\
Partial spleen infarction &
\end{tabular}

usually the staple line, injury of the spleen and liver, or trocar site bleeding. In order to avoid postoperative bleeding (POB), it is suggested that the blood pressure be maintained at the level of $140-150 \mathrm{~mm} \mathrm{Hg}$ at the final stage of the surgery and that the staple line be reinforced by sutures [18]. Zafar et al. conducted the study analysing factors influencing POB. Their study supports the thesis that oversewing the staple line reduces the incidence of POB [19]. Management of bleeding includes blood transfusion and revision surgery in order to localize the source of bleeding (Table II).

Leakage after LSG is a life-threatening complication that occurs in 1-3\% of patients undergoing primary surgery, and up to $10 \%$ in patients after revision procedure [20]. Leaks may occur anywhere along the staple line; however, in $85 \%$ of cases they form just below the gastroesophageal junction, which is probably associated with the increased intragastric pressure due to impaired peristalsis and ischaemia [21]. The occurrence of leaks after LSG is associated not only with the modification of surgical technique (oversewing the staple line, distance from the pylorus to the beginning of the stomach resection, size of the gastric tube) but also with the coexistence of the components of metabolic syndrome, mainly type 2 diabetes [22]. The multicentre study conducted by Benedix et al. analysed the medical histories of 5400 patients who had undergone the LSG. It confirmed that male gender and BMI $50-50.9 \mathrm{~kg} / \mathrm{m}^{2}$ are associated with significantly higher leak rate ( 2.5 vs. $1.6 \%, p=0.02$ and $p<0.01$ ) [23]. It has also been proven that smoking, improper postoperative diet, use of corticosteroids, and immunosuppressive treatment increase the risk of developing fistulas and leaks [24].

Patient may be totally asymptomatic or present with symptoms of septic shock, such as fever, abdominal pain, tachycardia, and peritonitis. Laboratory studies usually show leukocytosis, and elevated C-reactive protein and procalcitonin levels. Contrast-enhanced computed tomography is considered to be the most suitable diagnostic tool for the detection and confirmation of gastric leak. The management of patients with gastric leak is dependent on the condition of the patient. Conservative treatment may be introduced for patients in a stable condition, and it includes withholding food and fluids, intravenous hydration, administration of broad-spectrum antibiotics, and proton pump inhibitors. Lack of clinical and radiological improvement after conservative treatment is an indication for endoscopic intervention (Endoclips or endoprosthesis). If a patient is unstable, laparoscopic or open lavage and drainage of peritoneal cavity should be performed [20] (Tables III, IV). 
Table II. The incidence of postoperative bleeding after LSG based on modification of operating technique [19]

\begin{tabular}{|c|c|c|c|c|c|}
\hline Variable & Category & $\begin{array}{c}\text { No postoperative bleeding } \\
(n=97519)\end{array}$ & $\begin{array}{c}\text { Postoperative } \\
\text { bleeding }(n=623)\end{array}$ & Total $(n=98142)$ & $P$-value \\
\hline \multirow[t]{3}{*}{ Bougie size } & $<38 \mathrm{Fr}$ & $54,066(55.4)$ & $341(54.7)$ & $54,407(55.4)$ & 0.302 \\
\hline & $\geq 38 \mathrm{Fr}$ & $39,212(40.2)$ & $247(39.7)$ & $39,459(40.2)$ & \\
\hline & Missing & $4241(4.4)$ & $35(5.6)$ & $4276(4.4)$ & \\
\hline \multirow{4}{*}{$\begin{array}{l}\text { Staple line } \\
\text { treatment }\end{array}$} & No treatment & $22,420(23.0)$ & $181(29.1)$ & $22,601(23.0)$ & 0.005 \\
\hline & Oversew alone & $9864(10.1)$ & $68(10.9)$ & $9932(10.1)$ & \\
\hline & Buttress & $52,654(54.0)$ & $304(48.8)$ & $52,958(54.0)$ & \\
\hline & $\begin{array}{l}\text { Combination } \\
\text { of buttress and } \\
\text { oversew }\end{array}$ & $12,576(12.9)$ & $70(11.2)$ & $12,646(12.9)$ & \\
\hline
\end{tabular}

Table III. The incidence of leak and bleeding according to the reinforcement technique of the staple line

\begin{tabular}{|c|c|c|c|c|c|}
\hline Reinforcement technique & Number of patients & Leak & $\%$ & Bleeding & $\%$ \\
\hline No reinforcement & 189 & 9 & 4.8 & 26 & 13.7 \\
\hline Oversewing & 476 & 14 & 3.0 & 7 & 1.4 \\
\hline Peri-StripsDry & 312 & 1 & 0.3 & 0 & 0 \\
\hline Duet TRS & 76 & 6 & 7.8 & 1 & 1.3 \\
\hline SeamGuard & 63 & 2 & 3.2 & 1 & 1.6 \\
\hline Floseal & 46 & 1 & 2.2 & 0 & 0 \\
\hline
\end{tabular}

Table IV. The leak rate according to bougie size, distance from the pylorus, and staple line buttress [22]

\begin{tabular}{lccc} 
Variable & Number of patients & Number of leaks & $\%$ \\
\hline Bougie size $\geq 50$ & 377 & 3 & 0.9 \\
\hline Bougie size 40-49 Fr & 2394 & 41 & 1.7 \\
\hline Bougie size $<40 \mathrm{Fr}$ & 6152 & 153 & 2.5 \\
\hline Buttressing & 4780 & 102 & 2.1 \\
\hline No buttressing & 1143 & 37 & 3.2 \\
\hline Distance from the pylorus $<5 \mathrm{~cm}$ & 2500 & 49 & 2.0 \\
\hline Distance from the pylorus $\geq 5 \mathrm{~cm}$ & 5380 & 122 & 2.3
\end{tabular}

The main consequence of leak formation is the development of fistulas and abscesses. The diagnostic management includes X-ray examination of the upper gastrointestinal tract with contrast. However, in many cases the obtained results may be correct, especially when the leakage is located in the upper part of the left stomach due to the rapid passage of the contrast [25]. The leakage is usually visible across the staple line or in the left subdiaphragmatic region [26].

Another early complication that may occur after bariatric surgery is acute pancreatitis. Kumaravel et al. conducted a cohort study of all patients who underwent bariatric procedures, and they calculated the incidence of acute pancreatitis at $1.04 \%$ [27]. Patients usually report epigastric pain radiating to the back, nausea, vomiting, and fever. The development of acute pancreatitis after bariatric surgery is probably associated with the manipulation of peripancreatic tissue intraoperatively. Secondarily, it may be related to the impaired pancreatic microcirculation after gastrectomy [28]. The diagnosis is made based on computed tomography. Fluid replacement, optimization of electrolyte balance, antibiotics administration, and proper nutrition are essential points of initial management of acute pancreatitis.

Postoperative venous thromboembolism (VTE) is a life-threating complication after bariatric surgery, and it is one of the leading causes of early mortality. The incidence of VTE after bariatric surgery ranges from $0.06 \%$ to $2.20 \%$ [29]. Gambhir et al. proved that prolonged operative time, transfusions, and history of deep vein thrombosis (DVT) are associated with a higher risk of developing DVT or pulmonary embolism (PE) [30]. 
The cardinal signs of DVT include asymmetrical swelling, warmth, and pain in the lower extremity. Symptoms of pulmonary embolism are non-specific and may be present in other conditions. Characteristics signs of PE include tachycardia, chest pain, dyspnoea, and hypoxaemia. D-dimer levels should be measured in all patients suspected of developing DVT or PE, because it has a high negative predictive value [31]. The first-line imaging method for DVT is ultrasonography, and for PE - computed tomographic angiography. Anticoagulation is an essential point of both prophylaxis and treatment of thromboembolic events.

\section{Late complications}

The incidence of gastric stenosis after LSG is approximately $1 \%$, and this significantly increases in cases of revision surgery, to as much as $10 \%$ [32]. Patients present symptoms of food intolerance, dysphagia, nausea, and vomiting. An organic stenosis is associated with the existence of leak and abscess, overly tight gastric sleeve, mediastinal migration of the cardia, and intramural haematoma after oversewing the staple line. The causes of functional gastric stenosis include obstruction at the incisura angularis, axial torsion of the gastric tube, and small intestine volvulus [32]. The first-choice treatment of gastric stenosis is endoscopic pneumatic dilation, which is considered to be a safe and effective method.

Laparoscopic sleeve gastrectomy is also associated with the development or worsening of gastroesophageal reflux disease (GERD). Althuwaini et al. conducted research that included 213 patients who had undergone LSG. New-onset heartburn was reported in $47.06 \%$ of the cohort. The incidence of dysphagia and regurgitation also increased postoperatively [33]. Increased prevalence of GERD after LSG is associated with reduced tension of the lower oesophageal sphincter, blunting of the angle of His, decreased gastric emptying, and reduced gastric volume and compliance, which leads to increased intragastric pressure [34].

Nutritional deficiency is a condition that occurs as a late complication after LSG. A study conducted by Gehrer et al. showed the following deficits of microelements and vitamins: zinc (34\%), vitamin $D_{3}(32 \%)$, iron $(18 \%)$, vitamin $B_{12}(18 \%)$, and folic acid (22\%). However, the frequency of nutrient deficiencies was lower after LSG when compared with LRYGB [35]. Routine blood tests after LSG are recommended to detect vitamin deficits and to introduce appropriate supplementation.

\section{Conclusions}

Due to the constantly growing number of obese people around the world, the number of performed bariat- ric procedures has been rising accordingly. Laparoscopic sleeve gastrectomy, as with any kind of surgical intervention, is associated with the risk of postoperative complications. Adverse events associated with bariatric surgery are associated with the obesity itself, systemic disorders, and obesity-related diseases. It is important to be aware of the possible postoperative complications and to choose the correct surgical technique. Surgeons should monitor patients postoperatively to quickly recognize the occurrence of postoperative complications and to introduce appropriate methods of diagnosis and treatment.

\section{Conflict of interest}

The authors declare no conflict of interest.

\section{References}

1. Online source: https://www.who.int/en/news-room/factsheets/detail/obesity-and-overweight Access: 15.03.2020.

2. Han TS, Lean ME. A clinical perspective of obesity, metabolic syndrome and cardiovascular disease. JRSM Cardiovasc Dis 2016; 5: 2048004016633371.

3. Wojciak P, Pawluszewicz P, Diemieszczyk I, et al. Laparoscopic sleeve gastrectomy: a study of efficiency in treatment of metabolic syndrome components, comorbidities and influence on certain biochemical markers. Videosurgery Miniinv 2020; 15: 136-47.

4. Isoma B, Almgren P, Tuomi T, et al. Cardiovascular morbidity and mortality associated with the metabolic syndrome. Diabetes Care 2001; 24: 683-9.

5. Lakka HM, Laaksonen DE, Lakka TA, et al. The metabolic syndrome and total and cardiovascular disease mortality in middle-aged men. JAMA 2002; 288: 2709-16.

6. Welbourn R, Hollyman M, Kinsman R, et al. Bariatric surgery worldwide: baseline demographic description and one-year outcomes from the Fourth IFSO Global Registry Report 2018. Obes Surg 2019; 29: 782-95.

7. Alizadeh RF, Li S, Gambhir S, et al. Laparoscopic sleeve gastrectomy or laparoscopic gastric bypass for patients with metabolic syndrome: an MBSAQIP analysis. Am Surg 2019; 85: 1108-12.

8. Hoyuela C. Five-year outcomes of laparoscopic sleeve gastrectomy as a primary procedure for morbid obesity: a prospective study. World I Gastrointest Surg 2017; 9: 109-17.

9. Wojciak P, Pawłuszewicz P, Diemieszczyk I, et al. Laparoscopic sleeve gastrectomy: a study of efficiency in treatment of metabolic syndrome components, comorbidities and influence on certain biochemical markers. Videosurgery Miniinv 2020; 15: 136-47.

10. Marceau P, Hould FS, Simard S, et al. Biliopancreatic diversion with duodenal switch. World J Surg 1998; 22: 947-54.

11. Karmali S, Schauer P, Birch D, et al. Laparoscopic sleeve gastrectomy: an innovative new tool in the battle against the obesity epidemic in Canada. Can J Surg 2010; 53: 126-32.

12. Stefura T, Kacprzyk A, Droś J, et al. The hundred most frequently cited studies on sleeve gastrectomy. Videosurgery Miniinv 2020; 15: 249-67. 
13. Moulla Y, Lyros O, Blüher M, et al. Feasibility and aafety of bariatric surgery in high-risk patients: a single-center experience. J Obes 2018; 2018: 7498258.

14. Hajer AA, Wolff S, Benedic F, et al. Trends in early morbidity and mortality after sleeve gastrectomy in patients over 60 years: retrospective review and data analysis of the German Bariatric Surgery Registry. Obes Surg 2018; 28: 1831-7.

15. Chivot C, Robert B, Lafaye N, et al. Laparoscopic sleeve gastrectomy: imaging of normal anatomic features and postoperative gastrointestinal complications. Diagn Interv Imaging 2013; 94: 823-34.

16. Zellmer JD, Mathiason MA, Kallies KJ, et al. Is laparoscopic sleeve gastrectomy a lower risk bariatric procedure compared with laparoscopic Roux-en-Y gastric bypass? A meta-analysis. Am J Surg 2014; 208: 903-10.

17. Shikora SA, Mahoney CB. Clinical benefit of gastric staple line reinforcement (SLR) in gastrointestinal surgery: a meta-analysis. Obes Surg 2015; 25: 1133-41.

18. Sroka G, Milevski D, Shteinberg D, et al. Minimizing hemorrhagic complications in laparoscopic sleeve gastrectomy a randomized controlled trial. Obes Surg 2015; 25: 1577-83.

19. Zafar SN, Felton J, Miller K, et al. Staple line treatment and bleeding after laparoscopic sleeve gastrectomy. JSLS 2018; 22: e2018.00056.

20. Abou Rached A, Basile M, El Masri H. Gastric leaks post sleeve gastrectomy: review of its prevention and management. World J Gastroenterol 2014; 20: 13904-10.

21. D'Ugo S, Gentileschi P, Benavoli D, et al. Comparative use of different techniques for leak and bleeding prevention during laparoscopic sleeve gastrectomy: a multicenter study. Surg Obes Relat Dis 2014; 10: 450-4.

22. Parikh M, Issa R, McCrillis A, et al. Surgical strategies that may decrease leak after laparoscopic sleeve gastrectomy: a systematic review and meta-analysis of 9991 cases. Ann Surg 2013; 257: 231-7.

23. Benedix F, Benedix DD, Knoll C, et al. Are there risk factors that increase the rate of staple line leakage in patients undergoing primary sleeve gastrectomy for morbid obesity? Obes Surg 2014; 24: 1610-6.

24. Alizadeh RF, Li S, Inaba C, et al. Risk factors for gastrointestinal leak after bariatric surgery: MBASQIP analysis. J Am Coll Surg 2018; 227: 135-41.

25. Brockmeyer JR, Simon TE, Jacob RK, et al. Upper gastrointestinal swallow study following bariatric surgery: institutional review and review of the literature. Obes Surg 2012; 22: 1039-43.

26. Kim KW, Choi BI, Han JK, et al. Postoperative anatomic and pathologic findings at CT following gastrectomy. Radiographics 2002; 22: 323-36.

27. Kumaravel A, Zelisko A, Schauer P, et al. Acute pancreatitis in patients after bariatric surgery: incidence, outcomes, and risk factors. Obes Surg 2014; 24: 2025-30.

28. Sarwal A, Khullar R, Sharma A, et al. Acute pancreatitis as an unusual early post-operative complication following laparoscopic sleeve gastrectomy. J Minim Access Surg 2018; 14 164-7.

29. Aminian A, Andalib A, Khorgami Z, et al. Who should get extended thromboprophylaxis after bariatric surgery? A risk as- sessment tool to guide indications for post-discharge pharmacoprophylaxis. Ann Surg 2017; 265: 143-50.

30. Gambhir S, Inaba CS, Alizadeh RF, et al. Venous thromboembolism risk for the contemporary bariatric surgeon. Surg Endosc 2020; 34: 3521-6.

31. Stone J, Hangge P, Albadawi $\mathrm{H}$, et al. Deep vein thrombosis: pathogenesis, diagnosis, and medical management. Cardiovasc Diagn Ther 2017; 7 (Suppl 3): S276-84.

32. Turcu F, Balahura C, Doras I, et al. Symptomatic stenosis after laparoscopic sleeve gastrectomy - incidence and management in a high-volume bariatric surgery center. Chirurgia 2018; 113 : 826-36.

33. Althuwaini S, Bamehriz F, Aldohayan A, et al. Prevalence and predictors of gastroesophageal reflux disease after laparoscopic sleeve gastrectomy. Obes Surg 2018; 28: 916-22.

34. Laffin M, Chau J, Gill RS, et al. Sleeve gastrectomy and gastroesophageal reflux disease. J Obes 2013; 2013: 741097.

35. Gehrer S, Kern B, Peters T, et al. Fewer nutrient deficiencies after laparoscopic sleeve gastrectomy (LSG) than after laparoscopic Roux-Y-gastric bypass (LRYGB) - a prospective study. Obes Surg 2010; 20: 447-53.

Received: 27.03.2020

Accepted: 6.05.2020 